\title{
Electroacupuncture for older adults with mild cognitive impairment: study protocol for a randomized controlled trial
}

Albert Wing Nang Leung ${ }^{1 *}$, Linda Chiu Wa Lam², Andrew Ka Lun Kwan ${ }^{1}$, Celia Lai Lin Tsang ${ }^{1}$, Hong Wei Zhang ${ }^{1}$, Yuan Qi Guo ${ }^{1}$ and Chuan Shan Xu, , $^{*}$

\begin{abstract}
Background: Mild cognitive impairment is an intermediary state between normal aging and clinical Alzheimer's disease. Early intervention of mild cognitive impairment may be an important strategy in the management of Alzheimer's disease. The proposal aims to evaluate if electroacupuncture would optimize cognitive function in subjects with mild cognitive impairment and understand the role of electroacupuncture in the treatment of Alzheimer's disease.
\end{abstract}

Methods/design: A randomised patient- and assessor-blind sham-controlled trial is designed to assess whether electroacupuncture intervention decreases the rate of cognitive decline amongst older adults with mild cognitive impairment. One hundred and fifty subjects aged 65 years of age or over with a diagnosis of mild cognitive impairment are recruited from the community and elderly centre in Hong Kong. All subjects are randomly allocated into two groups (75 subjects each group): the electroacupuncture group and sham control. Participants in the electroacupuncture group receive electroacupuncture stimulation by sterile, disposable acupuncture needles inserted to the acupoints with a depth of 1 to $3 \mathrm{~cm}$. The acupuncture needles are subjected to $2 \mathrm{~Hz}$ electroacupuncture wihtat an intensity of 5 to $10 \mathrm{~mA}$. Each participant receives electroacupuncture for 8 weeks (once a day, 3 days a week) and the treatment lasts for 30 minutes each time. For sham electroacupuncture, needles are inserted to a depth of 1 to $2 \mathrm{~mm}$, and connected to the electroacupuncture device without any current passing through. Outcome measures (including primary and secondary outcome measures) are collected at baseline, at the end day of intervention, and months 4 and 6 after intervention. The primary outcome is measured by the Alzheimer Disease Assessment Scale-Cognitive subscale. Secondary outcomes are measured by the mini-mental state examination, category fluency text and the Short Form 12.

Discussion: The study will provide evidence for evaluating and understanding the role of electroacupuncture in the treatment of Alzheimer's disease.

Trial registration: This trial is registered with chictr.org (registration number: ChiCTR-TRC-12002414. Registration date: 11 August 2012.

Keywords: Electroacupuncture, Mild cognitive impairment, Alzheimer's disease, Cognitive function

\footnotetext{
*Correspondence: awnleung@cuhk.edu.hk; csxu@cuhk.edu.hk

'School of Chinese Medicine, Faculty of Medicine, The Chinese University of

Hong Kong, Shatin, Hong Kong

${ }^{3}$ Shenzhen Research Institute, The Chinese University of Hong Kong,

Shenzhen, China

Full list of author information is available at the end of the article
} 


\section{Background}

Nowadays, nearly 25 million people have been diagnosed with dementia worldwide, and the number is increasing by 5 million each year [1]. Alzheimer's disease (AD) is a common dementia in older people. Patients with AD usually suffer from a progressive decline in their cognition and functional ability $[2,3]$. These mental disorders seriously affect the quality of daily life of patients with $\mathrm{AD}$. As the Hong Kong population has aged rapidly in recent years, $\mathrm{AD}$ has become an important public health problem in Hong Kong $[4,5]$.

The current strategy mainly relies on complementary medicine for improving quality of life and delaying cognitive decline of patients with AD. However, there is as yet no really satisfactory treatment. It is well known that $\mathrm{AD}$ is a progressively cognitive decline, and mild cognitive impairment $(\mathrm{MCI})$ is an intermediary state between normal aging and clinical $\mathrm{AD}[6,7]$. The older adults with $\mathrm{MCI}$ are at increased risk for conversion to clinical $\mathrm{AD}[2,6,7]$. Emerging evidence shows that early improvement of MCI is very beneficial to decrease the prevalence of $\mathrm{AD}$ [8]. Thus, there is an urgent need to search novel and effective approaches for treating MCI.

Acupuncture is a common technique for treating neuropathy in traditional Chinese medicine. Manual and electrical stimulation on the acupoints are commonly used stimulation modes of acupuncture [9]. Increasing evidence shows that electroacupuncture could reduce the functional deficits of neurophathy [10] and prevent cognitive deficiency involved in brain diseases [11]. Jing and colleagues [12] showed that electroacupuncture could significantly improve learning and memory capacity. In mainland China, acupuncture has been used as an alternative medicine for $\mathrm{AD}$ and is claimed to be effective in improving intelligence. Recently, Xue and colleagues $[13,14]$ reported that electroacupuncture may effectively improve the learning-memory capacity of amyloid precursor protein transgenic $\mathrm{AD}$ mice and alleviate the pathological changes in neurons of the hippocampal region. Zhao and colleagues [15] showed that electroacupunture could shorten the waiting time for recognition and decrease the psychological stress. Many relevant clinical studies of electroacupuncture for dementia have been carried out in mainland China and benefit was reported in up to 70 to $90 \%$ of the treatment group, but a review from the Cochrane Collaboration demonstrated that true randomised controlled trials and high-quality trials are non-existent $[16,17]$. Randomised controlled trials are urgently needed.

\section{Objective}

The primary objective of this study is to evaluate if electroacupuncture can optimize cognitive function in subjects with $\mathrm{MCI}$, and to understand the role of electroacupuncture in the treatment of $\mathrm{AD}$.

\section{Methods/design}

\section{Study design}

The study is a randomised, patient- and assessor-blind sham-controlled trial with a 1:1 allocation ratio. The subjects $(\mathrm{n}=150)$ are randomly allocated to the electroacupuncture group $(\mathrm{n}=75)$ and sham control group $(\mathrm{n}=75)$. This project is approved by the Joint Chinese University of Hong Kong-New Territories East Cluster Clinical Research Ethics Committee (CRE-2011.515-T). Written informed consent is obtained from each participant. The design is described in Fig. 1.

\section{Participants}

Subjects who aged 65 years of age or over with a diagnosis of MCI are recruited from the community and elderly centre in Hong Kong.

\section{Inclusion criteria}

All subjects are assessed using the Clinical Dementia Rating. Case definition of MCI is based on Peterson's criteria. In this project, the eligible criteria are adopted following the methods described by Lam and colleagues $[5,18]$. The participants should be willing and able to travel to our treatment sites.

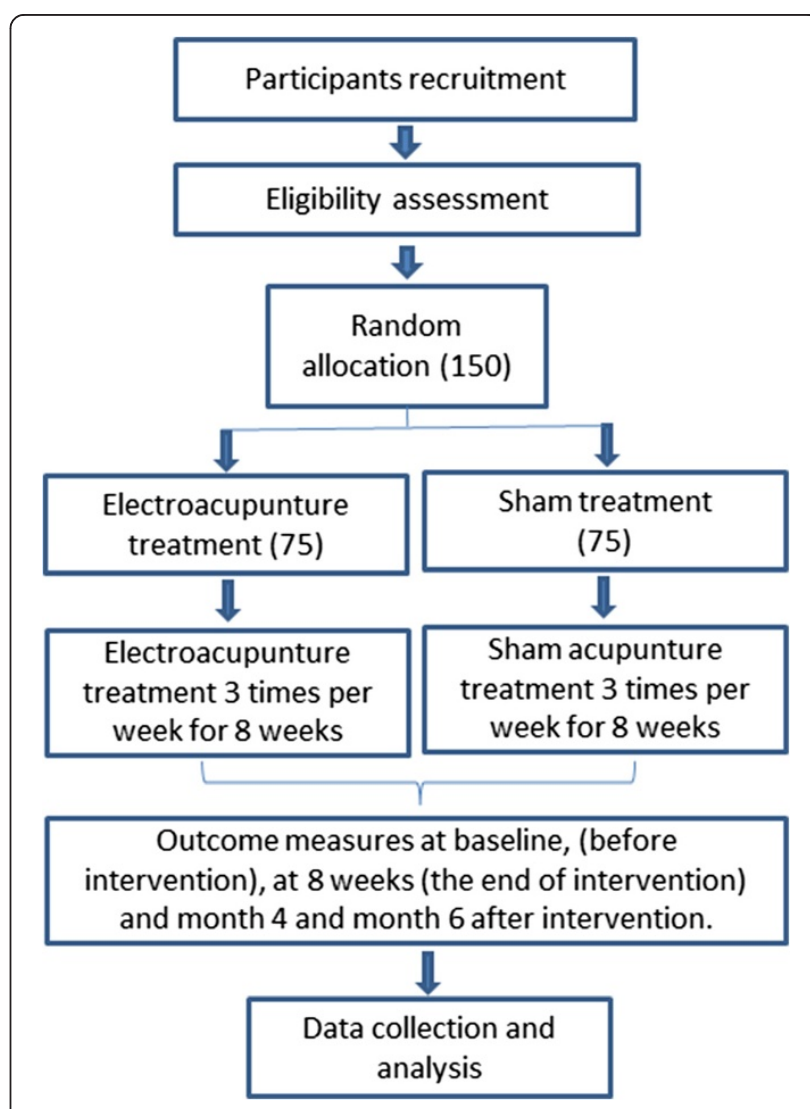

Fig. 1 Study flow chart 


\section{Exclusion criteria}

The exclusion criteria are as follows: dementia, $\mathrm{AD}$, notable cognitive impairment (score of 23 or less as evidenced by mini-mental state examination (MMSE), current psychiatric disorders (for example, depression, anxiety), history of alcohol or drug abuse, bleeding disorder, currently undergoing acupuncture/taking drug for cognitive problems, or currently treated with medications affecting cognition or other serious illnesses (for example, clinically significant active heart, renal, or hepatic diseases). Patients who have difficulty in reading and understanding study questionnaire are also excluded.

\section{Interventions}

Participants randomised to the electroacupuncture group will receive electroacupuncture stimulation after baseline assessment. The electroacupuncture treatment is carried out by a Chinese Medicine Practitioner registered with the Chinese Medicine Council of Hong Kong, who has at least 3 years of clinical acupuncture experience. From the view of Chinese medicine theory, the deficiency of the kidney is the main pathogenesis of $\mathrm{MCI}$; thus, each participant will receive electroacupuncture at traditional acupuncture points: EX-HN1 (Sishencong); GV20 (Baihui); GB13 (Benshen); GB20 (Fengchi); GB39 (Xuanzhong); DU24 (Shenting); KI3 (Taixi) $[19,20]$. The rationale for choosing the acupoints is as follows: the acupoints on the head such as EX-HN1 (Sishencong), GV20 (Baihui), GB13 (Benshen), DU24 (Shenting), and GB20 (Fengchi) have the local effect of regulating the brain function; the other acupoints on the lower limb, such as GB39 (Xuanzhong), and KI3 (Taixi), have the effect of tonifying the kidney $[19,20]$. Sterile, disposable acupuncture needles with a diameter of $0.20 \mathrm{~mm}$ are used in the study. The puncture is made in accordance with the standards of traditional Chinese medicine, to a depth of 1 to $3 \mathrm{~cm}$ depending on the thickness of the local tissues. The acupoints are subjected to 2 $\mathrm{Hz}$ electroacupuncture from an electroacupuncture device (Hwato, Electronic Acupuncture Treatment Instrument, Suzhou Medical Appliance Factory, Jiangsu, China) at an intensity (5 to $10 \mathrm{~mA}$ ) that can produce a muscle twitch acceptable to the participant. Each participant in the electroacupuncture group receives electroacupuncture for 8 weeks (once a day, 3 days a week) and the treatment lasts for 30 minutes each time $[19,20]$.

Participants randomised to the sham control group receive sham electroacupuncture treatment. For sham electroacupuncture, the short acupuncture needle is inserted to a depth of 1 to $2 \mathrm{~mm}$, which is deep enough to make the acupuncture needle stand up on the skin. The needles are only connected to the electroacupuncture device without any current passing through [21,22]. Other treatment procedures are similar to those of the electroacupuncture group.
All participants are advised not to seek any other alternative treatment affecting cognitive function (including any traditional Chinese medicine treatment or laser acupuncture, or moxibustion or other physiotherapies) during the trial period. Treatment compliance is also monitored at each appointment.

\section{Randomisation}

Participants are randomly allocated to either the electroacupuncture group or sham control group after the baseline assessment. The randomisation is performed according to computer generated random allocation treatment codes. The randomisation list is kept by an independent investigator not involving in the care or assessment of the participants, or in the data collection and analysis.

\section{Blinding}

All participants receive similar treatment procedures. All investigators who administer the outcome measurement questionnaire and statistician are blinded to the randomisation status. In order to blind the patients, we also tell them that the electric stimulation is rather mild and some may have no obvious sensation during treatment.

\section{Primary outcome}

Cognitive decline is measured using the Chinese version of the Alzheimer Disease Assessment Scale-Cognitive subscale (ADAS-cog) [13]. The ADAS-cog is sensitive to change over time, including some subscales for testing list learning, object naming, commands, ideational apraxia, construction, orientation, and recognition. The total possible score is 70. A score increase indicates greater severity of impairment.

The ADAS-cog is assessed at baseline (before intervention), at 8 weeks (the end of intervention) and months 4 and 6 after intervention.

\section{Secondary outcome}

Secondary measures include the MMSE, category fluency test (CFT) and the Short Form 12 (SF12) [2, 18, 23].

The MMSE is an 11-question measure that tests five areas of cognitive function (orientation, registration, attention and calculation, recall, and language). The maximum score is 30 and a score below 24 is considered abnormal for dementia screening [2].

The CFT is used for evaluating patient executive function, semantic memory and language. The CFT requires the subjects to generate exemplars in three categories for 1 minute per category as described by Lam and colleagues [18].

The SF12 includes 12 items testing eight concepts of both physical and mental health. The physical and mental component summary scales are scored using norm-based methods [23]. 
The MMSE, CFT, SF12 are also assessed at baseline (before intervention), at 8 weeks (the end of intervention) and months 4 and 6 after intervention.

\section{Other outcomes}

Adverse events are assessed by a Chinese Medicine Practitioner at each session of acupuncture. If the participants feel discomfort, such as vomiting, nausea, dizziness, or dry mouth or itching after acupuncture, these events are recorded and assessed at the end of treatment [24]. Additionally, the use of other any additional treatment or drugs must be recorded during follow-up.

\section{Sample size}

Base on previous related studies [25-27], we assume the standard deviation of ADAS-cog to be 6. To detect a 3point change on the scale of ADAS-cog between the electroacupuncture and sham treatment groups, a sample size of 126 will be needed with a false-positive error of $5 \%$ and power of $80 \%$. Since an overall dropout rate of $20 \%$ was anticipated, the required sample size will be 150 (75 patients per group).

\section{Data analysis}

The differences between electroacupuncture and sham treatment group are assessed by an analysis of covariance using baseline score as a covariate for the primary variables. Descriptive statistics and an analysis of covariance in the presence of baseline covariates are conducted for the secondary variables. A generalized linear model is used to adjust for possible confounding variables such as age, sex, social class, and so forth. Categorical data are analysed by means of chi-squared tests. A non-parametric approach (Mann-Whitney test) is used to analyse the subscales and interval data. Frequency tables and charts are used to analyse questions in the adverse events questionnaire. All statistical analyses are performed using the Statistical Package for Social Sciences (SPSS, SPSS Inc., Chicago, USA) version 16.0. A two-sided $P$-value of 0.05 or less is considered to be significant.

\section{Discussion}

Current management of $\mathrm{AD}$ mainly takes a comprehensive approach, including pharmacological and non-pharmacological interventions for cognitive and neuropsychiatric symptoms. MCI is considered an at-risk state for further cognitive deterioration. At present, there is no drug approved for the prevention of cognitive decline in people with $\mathrm{MCI}$. The present study is designed to evaluate if electroacupuncture can optimize cognitive function in subjects with $\mathrm{MCI}$ and to understand the role of electroacupuncture in the treatment of Alzheimer's disease.

A successful blinding during the trial will help maintain the comparability between groups. All investigators who administer the outcome measurement questionnaire and statistician are blinded to the randomisation status. We will also take measures to blind the patients. A conventional treatment is given to patients in both groups. Although we could not blind the acupuncturists, detailed instruction, such as how to avoid unnecessary conversation, to minimise the possible patient-practitioner interaction is given to them. When the acupuncturists withdraw the sham needle, they are also instructed to block the patients' line of sight with their back or hand. The advice given to patients not to seek other treatment aims also to help maintain the comparability between groups.

Allocation concealment methods seem to adequately guard against bias. In the trial, a colleague who is not involved in the baseline assessment is responsible for preparing the sequentially numbered, opaque, sealed envelopes that contain the intervention group assignment based on the random list generated by the computer program. After the eligible patients enter the trial, the acupuncturist will open the envelopes to find the intervention assignment.

In the acupuncture clinic, most acupuncturists individualise their interventions based on the constitutional and traditional Chinese medicine diagnosis of the individual presenting with symptoms. Considering that a formulaic, fixed acupuncture protocol is relatively easy to generalize to different regions worldwide and to decrease study bias, we chose uniform acupoints for all the participants in the trial. Additionally, the sham control intervention we used in the trial is really called minimal acupuncture or superficial needling, which has been often used as a sham control in clinical acupuncture trials in the last 20 years [21]. For the study of the efficacy of electroacupucnture, a sham acupuncture in the form of superficial needling may control for needle penetration [22]. Although a possible effect of superficial needling may exist, it is assumed to be rather small with a needling depth of 1 to $2 \mathrm{~mm}$ and without electrical stimulation compared to the normal acupuncture with a needling depth of 10 to $30 \mathrm{~mm}$ and electrical stimulation. It should be a better choice than nonpenetration needling as a control as we want to achieve a better blinding effect in the efficacy study.

At the end of this project, the preventative potential will be discussed in light of the results obtained. The findings of the project are expected to provide evidence for the efficacy of electroacupuncture treatment in improving cognitive function in people with $\mathrm{MCI}$.

\section{Trial status}

This trial is currently ongoing.

\section{Abbreviations}

AD: Alzheimer's disease; ADAS-cog: Alzheimer Disease Assessment Scale-Cognitive subscale; CFT: category fluency test; MCl: mild cognitive impairment; MMSE: mini-mental state examination; SF12: Short Form 12. 


\section{Competing interests}

The authors declare that they have no competing interests.

\section{Authors' contributions}

Conception and design, critical revision for this study protocol: AWNL, LCWL, YQG, HWZ, CSX. Recruiting subjects and assessment: AKLK, CLLT.

Acupuncture and data treatment: AKLK. All authors read and approved the final manuscript.

\section{Acknowledgements}

The project is supported by a grant from Health and Medical Research Fund (09100191) and the general research fund (GRF) grant from Hong Kong research grant committee (RGC) (476912). We would also like to express our sincere thanks to Ms Ada WT Fung and Mr Kelvin Wong Yuk Ming.

\section{Author details}

${ }^{1}$ School of Chinese Medicine, Faculty of Medicine, The Chinese University of Hong Kong, Shatin, Hong Kong. ${ }^{2}$ Department of Psychiatry, The Chinese University of Hong Kong, Shatin, Hong Kong. ${ }^{3}$ Shenzhen Research Institute, The Chinese University of Hong Kong, Shenzhen, China.

\section{Received: 2 February 2015 Accepted: 5 May 2015}

Published online: 27 May 2015

\section{References}

1. Gentile S. Second-generation antipsychotics in dementia: beyond safety concerns. A clinical, systematic review of efficacy data from randomised controlled trials. Psychopharmacology (Berl). 2010;212:119-29.

2. Vidovich MR, Lautenschlager NT, Flicker L, Clare L, Almeida OP. The PACE study: a randomised clinical trial of cognitive activity (CA) for older adults with mild cognitive impairment (MCl). Trials. 2009;10:114.

3. Workman B, Dickson F, Green S. Early dementia - optimal management in general practice. Aust Fam Physician. 2010;39:722-6.

4. Chan MC, Chong CS, Wu AY, Wong KC, Dunn EL, Tang OW, et al. Antipsychotics and risk of cerebrovascular events in treatment of behavioural and psychological symptoms of dementia in Hong Kong: a hospital-based, retrospective, cohort study. Int J Geriatr Psychiatry. 2010;25:362-70.

5. Chan WC, Lam LC, Tam CW, Lui WW, Chan SS, Chan WM, et al. Prevalence of neuropsychiatric symptoms in chinese older persons with mild cognitive impairment - a population-based study. Am J Geriatr Psychiatry. 2010;18:948-54.

6. Saxton J, Snitz BE, Lopez OL, Ives DG, Dunn LO, Fitzpatrick A, et al. GEM Study Investigators. Functional and cognitive criteria produce different rates of mild cognitive impairment and conversion to dementia. J Neurol Neurosurg Psychiatry. 2009;80:737-43.

7. Brooks LG, Loewenstein DA. Assessing the progression of mild cognitive impairment to Alzheimer's disease: current trends and future directions. Alzheimers Res Ther. 2010;2:28.

8. Levin OS, Yunishchenko NA, Dudarova MA. Efficacy of aktinol memantine in moderate cognitive impairments. Neurosci Behav Physiol. 2010;40:926-33.

9. Yeung W, Chung K, Leung Y, Zhang S, Law ACK. Traditional needle acupuncture treatment of insomnia: a systematic review of randomized controlled trials. Sleep Med. 2009;10:694-704.

10. Lin CC, Chen MC, Yu SN, Ju MS. Chronic electrical stimulation of four acupuncture points on rat diabetic neuropathy. Conf Proc IEEE Eng Med Biol Soc. 2005;4:4271-4.

11. Dos Santos Jr JG, Tabosa A, Do Monte FH, Blanco MM, De Oliveira Freire A, Mello LE. Electroacupuncture prevents cognitive deficits in pilocarpine-epileptic rats. Neurosci Lett. 2005;384:234-8.

12. Jing $X H$, Chen $S L$, Shi $H$, Cai $H$, Jin ZG. Electroacupuncture restores learning and memory impairment induced by both diabetes mellitus and cerebral ischemia in rats. Neurosci Lett. 2008;443:193-8.

13. Xue WG, Ge GL, Zhang Z, Xu H, Bai LM. Effect of electroacupuncture on the behavior and hippocampal ultrastructure in APP $695 \mathrm{~V} 717$ I transgenic mice. Zhen Ci Yan Jiu. 2009;34:309-14.

14. Xue WG, Zhang Z, Bai LM, Xu H, Wu HX. Effect of electroacupuncture on the behavior and the expression of amyloid beta-protein, amyloid precursor protein and ChAT in APP $695 \mathrm{~V} 717$ I transgenic mice. Zhen Ci Yan Jiu. 2009;34:152-8.
15. Zhao L, Zhang H, Zheng Z, Huang J. Electroacupuncture on the head points for improving gnosia in patients with vascular dementia. J Tradit Chin Med. 2009;29:29-34.

16. Lee MS, Shin BC, Ernst E. Acupuncture for Alzheimer's disease: a systematic review. Int J Clin Pract. 2009;63:874-9.

17. Peng WN, Zhao H, Liu ZS, Wang S. Acupuncture for vascular dementia. Cochrane Database Syst Rev. 2007;2:CD004987.

18. Lam LC, Tam CW, Lui W, Chan WC, Chan SS, Chiu HF, et al. Screening of mild cognitive impairment in Chinese older adults - a multistage validation of the Chinese abbreviated mild cognitive impairment test. Neuroepidemiology. 2008;30:6-12.

19. Liu XP, Liu ZY. Advances of acupuncture and moxibustion for cognitive impairment. Xinjiang J Tradit Chin Med. 2009;27:85-7.

20. Yang CZ, Zong J, Zhu AH. Study on the syndrome of senile minor cognitive injury. J Beijing Univ Tradit Chin Med (Clin Med). 2003;10:12-6.

21. Zhang HW, Tang JL. Design and choice of placebo acupuncture in clinical trial. Chin J Integr Tradit West Med. 2003;23:247-503.

22. White AR, Filshie J, Cummings TM. International Acupuncture Research Forum. Clinical trials of acupuncture: consensus recommendations for optimal treatment, sham controls and blinding. Complement Ther Med. 2001:9:237-45.

23. Van Uffelen JG, Hopman-Rock M, Chin A, Paw MJ, van Mechelen W. Protoco for Project FACT: a randomised controlled trial on the effect of a walking program and vitamin B supplementation on the rate of cognitive decline and psychosocial wellbeing in older adults with mild cognitive impairment [ISRCTN19227688]. BMC Geriatr. 2005;5:18.

24. Yang BF, Zeng XH, Liu Y, Fu QN, He T, He T, et al. Effect of acupuncture treatment on vascular cognitive impairment without dementia: study protocol for a randomized controlled trial. Trials. 2014;15:442-6.

25. Zhu YL, Jia JP. Clinical observation on catgut implantation at acupoint for treatment of Alzheimer's disease. Chin Acupunct Moxibustion. 2008;28:37-40.

26. de Jong $D$, Jansen $R$, Hoefnagels W, Jellesma-Eggenkamp M, Verbeek M, Borm $G$, et al. No effect of one-year treatment with indomethacin on Alzheimer's disease progression: a randomized controlled trial. PLoS One. 2008;3:e1475.

27. Rafii MS, Walsh S, Little JT, Behan K, Reynolds B, Ward C, et al. Alzheimer's disease cooperative study. A phase II trial of huperzine $A$ in mild to moderate. Alzheimer Dis Neurol. 2011;76:1389-94.

\section{Submit your next manuscript to BioMed Central and take full advantage of:}

- Convenient online submission

- Thorough peer review

- No space constraints or color figure charges

- Immediate publication on acceptance

- Inclusion in PubMed, CAS, Scopus and Google Scholar

- Research which is freely available for redistribution 